analyze trends over time and further compare disciplinary differences. RESULTS/ANTICIPATED RESULTS: UC is a diverse institution that includes world-renowned creative schools (the College Conservatory of Music and the College of Design, Architecture, Art, and Planning), as well as traditional colleges of medicine, nursing, pharmacy, allied health, engineering, business, arts and sciences, etc. UC also includes two branch campuses that specialize in associate's degree level education. Given the diversity in educational and research missions across these areas, we anticipate discovering several themes within the RPT guidelines, primarily centered around the traditional foundations of faculty work such as service, research, and teaching. We anticipate strong differences by college and disciplinary focus, with emphasis on collaborative work and engagement increasing as RPT guidelines become more current. DISCUSSION/ SIGNIFICANCE OF IMPACT: Our experience is that faculty members want to engage in collaborative work when possible and appropriate, but their perception is that independent contributions to their field are more highly valued than interdisciplinary work. As universities rush to endorse and promote interdisciplinary, team-oriented research and teaching, this study will afford a better understanding of the types of activities valued at one large and diverse urban institution, grounded in the actual language of RPT criteria.

3184

\section{Development of a Leadership Assessment Scale in Translational Science}

Roger Vaughan ${ }^{1}$, Rhonda G Kost, MD ${ }^{1}$, Donna Brassil ${ }^{1}$, Michelle Romanick ${ }^{1}$ and Barry S. Coller, MD ${ }^{1}$

${ }^{1}$ Rockefeller University

OBJECTIVES/SPECIFIC AIMS: To create the instrument, we employed a modified Delphi approach by conducting a thorough literature review on Leadership to help concretize the relevant constructs, and then usied these extracted constructs as a springboard for the Rockefeller Team Science Educators (TSE's) to discuss and refine the leadership domain areas, collectively creating domainspecific survey items, and then further discussed and refining the number, grouping, and wording of the items. METHODS/STUDY POPULATION: We piloted the Leadership Survey by having all of the Rockefeller TSEs rate Clinical Scholars. Each item was answered using a six-point Likert scale where a low score indicated poor expression of the specific leadership attribute and a high score represented excellent expression of the specific leadership attribute. RESULTS/ANTICIPATED RESULTS: Means, medians, standard deviations, and ranges of each item were calculated and tabulated. A complete (Pearson) correlation matrix was computed so that the raw inter-item relationships can be observed. For each a priori Domain an equal weighted summary scale was created and tabulated for review. The internal consistency of each a priori scale was assessed by calculating Cronbach's Alpha $(\alpha)$. Items with low Item to Construct coefficients were candidates for elimination or modification, and overall scales with low's will undergo further discussion. To challenge our assumptions of the construction and integrity of each domain, we employed exploratory Principal Components Analysis (PCA), followed by orthogonally rotated Factor Analysis (FA). We also forced the PCA / FA analysis to extract the a priori dimensions that allowed us to compare if the empirical and a priori structures match. DISCUSSION/SIGNIFICANCE OF IMPACT: We are partnering with the CTSA programs at Penn and Yale to assess issues of generalizability and scalability. We are working with Vanderbilt to install survey onto REDCap for ease of dissemination.
Will continue to assess psychometric properties and refine as we receive more input.

\section{5}

Diseased and Healthy Gastrointestinal Tissue Data Mining requires an Engaged Transdisciplinary team

Sana Syed ${ }^{1}$, Marium Naveed Khan ${ }^{1}$, Alexis Catalano ${ }^{1}$, Zambia Team², Pakistan Team ${ }^{3}$, Christopher Moskaluk ${ }^{1}$, Jason Papin ${ }^{1}$, S. Asad Ali ${ }^{3}$, Sean R. Moore ${ }^{1}$ and Donald E. Brown ${ }^{1}$ ${ }^{1}$ University of Virginia; ${ }^{2}$ Queen Mary University of London, University of Zambia and ${ }^{3}$ Aga Khan University

OBJECTIVES/SPECIFIC AIMS: To establish an effective team of researchers working towards developing and validating prognostic models employing use of image analyses and other numerical metadata to better understand pediatric undernutrition, and to learn how different approaches can be brought together collaboratively and efficiently. METHODS/STUDY POPULATION: Over the past 18 months we have established a transdisciplinary team spanning three countries and the Schools of Medicine, Engineering, Data Science and Global Health. We first identified two team leaders specifically a pediatric physician scientist (SS) and a data scientist/engineer (DB). The leaders worked together to recruit team members, with the understanding that different ideas are encouraged and will be used collaboratively to tackle the problem of pediatric undernutrition. The final data analytic and interpretative core team consisted of four data science students, two PhD students, an undergraduate biology major, a recent medical graduate, and a $\mathrm{PhD}$ research scientist. Additional collaborative members included faculty from Biomedical Engineering, the School of Medicine (Pediatrics and Pathology) along with international Global Health faculty from Pakistan and Zambia. We learned early on that it was important to understand what each of the member's motivation for contributing to the project was along with aligning that motivation with the overall goals of the team. This made us help prioritize team member tasks and streamline ideas. We also incorporated a mechanism of weekly (monthly/bimonthly for global partners) meetings with informal oral presentations which consisted of each member's current progress, thoughts and concerns, and next experimental goals. This method enabled team leaders to have a 3600 mechanism of feedback. Overall, we assessed the effectiveness of our team by two mechanisms: 1) ongoing team member feedback, including team leaders, and 2) progress of the research project. RESULTS/ANTICIPATED RESULTS: Our feedback has shown that on initial development of the team there was hesitance in communication due to the background diversity of our various member along with different cultural/social expectations. We used ice-breaking methods such as dedicated time for brief introductions, career directions, and life goals for each team member. We subsequently found that with the exception of one, all other team members noted our working environment professional and conducive to productivity. We also learnt from our method of ongoing constant feedback that at times, due to the complexity of different disciplines, some information was lost due to the difference in educational backgrounds. We have now employed new methods to relay information more effectively, with the use of not just sharing literature but also by explaining the content. The progress of our research project has varied over the past 4-6 months. There was a steep learning curve for almost every member, for example all the data science students had never studied anything related to medicine during their education, including minimal if none exposure to the ethics of medical research. Conversely, team members with medical/biology 